\title{
Giant Fibroadenoma-Radiologically Simulating Phyllodes Tumor: A Case Report of Unresolved Diagnostic Enigma
}

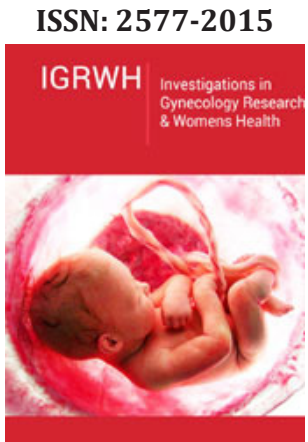

*Corresponding author: Jitendra Parmar,Department of Radiology, India

Submission: 眥 August 27, 2020

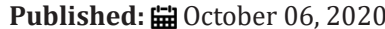

Volume 3 - Issue 4

How to cite this article: Jitendra Parmar, Tapan Patel, Sumita Choudhary, Anagha Zope, Maulik Vora, etal. Giant FibroadenomaRadiologically Simulating Phyllodes Tumor: A Case Report of Unresolved Diagnostic Enigma. 3(4). IGRWH. 000570. 2020.

DOI: 10.31031/IGRWH.2020.03.000570

Copyright@ Jitendra Parmar, This article is distributed under the terms of the Creative Commons Attribution 4.0 International License, which permits unrestricted use and redistribution provided that the original author and source are credited.

\section{Jitendra Parmar ${ }^{1 *}$, Tapan Patel $^{1}$, Sumita Choudhary ${ }^{1}$, Anagha Zope ${ }^{2}$, Maulik} Vora $^{3}$, Nishith Chaudhari ${ }^{1}$, Bhoomi Angirish ${ }^{1}$, Drashti Patel ${ }^{1}$

${ }^{1}$ Department of Radiology, Apollo Hospitals International Limited, Ahmedabad, India

${ }^{2}$ Department of Surgical Oncology, Apollo Hospitals International Limited and Apollo Comprehensive Blood \& Cancer Center, India

${ }^{3}$ Department of Radiology, Indira Gandhi Medical College, Shimla, India

\begin{abstract}
Fibroadenoma and phyllodes tumor are fibroepitheliallesions, thus may have similar histopathological appearance and share overlapping clinical as well as radiological features. The distinction between these two lesions is clinically important as both lesions have different management. There is substantial overlap between fibroadenomas and phyllodes tumors. We present a similar case report of a 23 years-old female patient with classical radiological features of phyllodes tumor, which turned out to be a giant type of fibroadenoma on histopathology after surgical excision.
\end{abstract}

Keywords: Giant fibroadenoma; Phyllodes; Mammography; Ultrasound; MRI

Abbreviations: BIRADS: Breast Imaging-Reporting and Data System

\section{Introduction}

Fibroadenoma is a benign lesion, accounting for well over half of breast tumors, while phyllodes tumor have spectrum of diseases ranging from benign to malignant lesions, accounting for $1 \%$ of all breast neoplasms [1]. Fibroadenoma and phyllodes tumor are fibroepithelial lesions [2], thus may have similar histopathological appearance and share overlapping clinical as well as radiological features $[3,4]$. The distinction between these two lesions is clinically important as the both lesions are managed differently. Fibroadenoma may be closely followed up or treated with simple enucleation, while phyllodes tumors must be surgically excised with wide excision and adequate margins to avoid local recurrence and subsequent surgery [5,6].

Most studies have reported differentiating fibroadenomas and phyllodes tumors radiologically on the basis of their shape and margins. While phyllodes tumours are lobulated in shape, fibroadenomas are frequently oval in shape with well circumscribed margins. Hypervascularity is often associated with phyllodes tumor, whereas fibroadenomas are hypo-vascular [3]. In spite of these well recognised and reported differentiating radiological features, there is a substantial imaging overlap between fibroadenomas and phyllodes tumors $[7,8]$. We present a similar case of a 23 years-old female patient with well reported radiological features of phyllodes tumor, which turned out to be a giant type of fibroadenoma on histopathology after surgical excision of the lesion.

\section{Case Report}

\section{Case history}

A 23-years-old female presented with a painless lump in right breast which was rapidly increasing in size over 2 months, since the time she noticed the swelling. She had no complaints of pain, skin changes, trauma or nipple discharge. The patient had no personal or family history of breast cancer. Her menstrual cycle was regular and was a nullipara with no history of any hormonal therapy.

\section{Examinations}

a) Physical Examination: The patient was well nourished with body mass index within normal range and stable vitals. 
b) Local examination: The right breast was asymmetrically enlarged with a large well defined, freely mobile, non-tender palpable lump. It was approximately $12 \times 10 \mathrm{~cm}$ in size predominantly situated in the lower outer, outer central and upper outer quadrants which was having a bosselated superior margin. There were no skin or nipple changes or increased temperature. Both axilla and left breast were unremarkable.

Routine laboratory investigations were within normal range

\section{Radiological Investigations:}

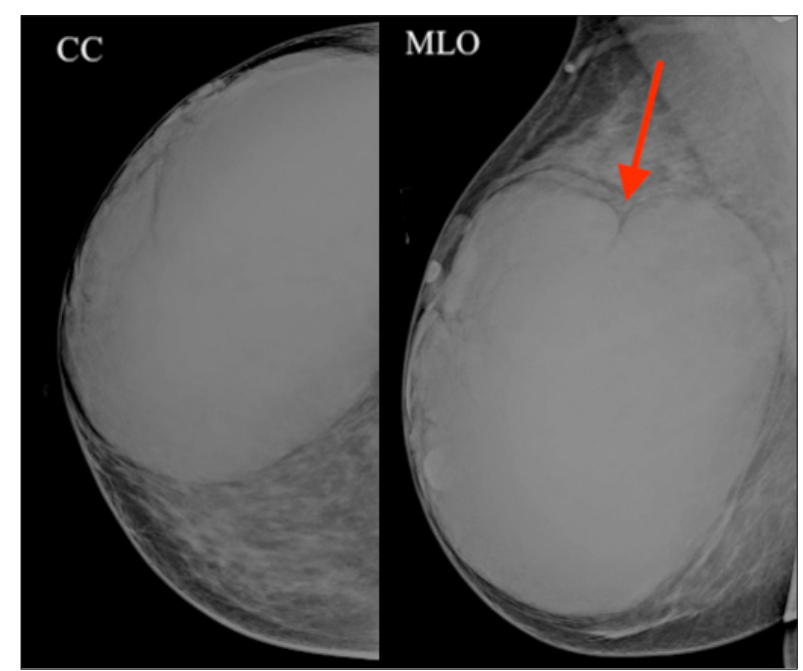

Figure 1: Cranio-caudal (Figure 1a) and Mediolateral-Oblique (Figure 1b) views of right breast showed a well-defined, oval shaped, high density mass lesion with well circumscribed margins and gentle lobulations at superior aspect (Red arrow in figure $1 \mathrm{~b})$.

a) Mammography (Figure 1): Cranio-caudal and Mediolateral-Oblique views of right breast were performed, which revealed a well-defined, oval shaped, high density mass lesion with well circumscribed margins and gentle lobulations at superior margins. No pleomorphic micro-calcifications or surrounding architectural distortion was seen. There were no skin thickening or no nipple retraction.

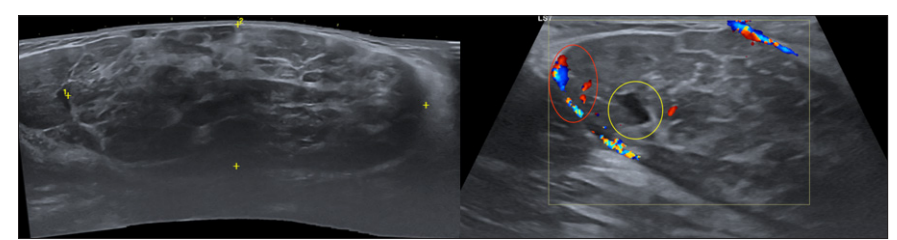

Figure 2: Panoramic image of right breast (Figure 2a) showed a large oval shaped well encapsulated isoechoic to hypoechoic mass lesion with internal echogenic strands. Color Doppler image (Figure $2 \mathrm{~b})$ revealed few cystic areas, suggestive of positive cleft sign (yellow circle) and significant perilesional and intralesional vascularity (red circle). b) Ultrasound (Figure 2): A large oval shaped isoechoic to hypoechoic well encapsulated mass lesion was seen in right breast, which measured $\sim 12 \times 11 \times 6 \mathrm{~cm}$ in size. It showed few eccentric cystic areas, suggestive of positive cleft sign. It also revealed multiple intralesional echogenic striations. On Color Doppler, it revealed significant perilesional and intralesional vascularity with multiple hypertrophied and tortuous vascular channels in surrounding breast parenchyma. The lesion was hard with high strain and elasticity ratio on elastography (not shown in figures).
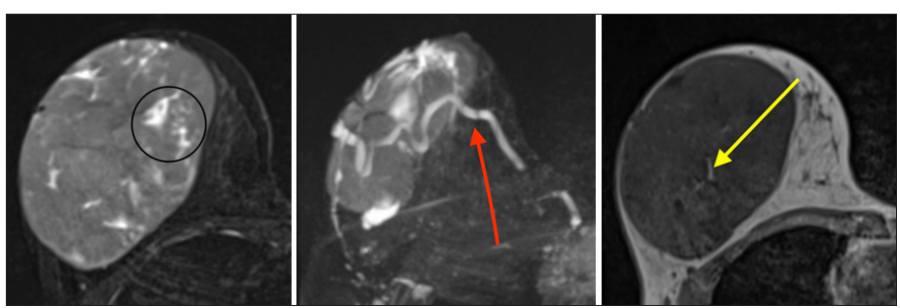

Figure 3: Magnetic Resonance Imaging; Axial T2WI DIXON (Figure $3 a$ and $3 b$ ) and Axial T1WI NONFATSAT (Figure 3c) confirmed the well-defined encapsulated mass lesion in right breast. It showed intralesional multiple linear hyperintense and hypointense areas, suggestive of vascular channels and fibrous septae respectively (Black Circle in Figure 3a). It also confirmed the eccentric cystic changes along inferior surface (Figure 3b) and gentle lobulations of superior surface. Few hypertrophied and tortuous vessels were also noted in the surrounding breast parenchyma (Red arrow in Figure $3 b)$. Non-FatSat T1WI sequence on Figure 3c showed lipomatous component (Yellow arrow).

c) Magnetic Resonance Imaging (Figure 3): MRI confirmed a well-defined encapsulated mass lesion in right breast, with eccentric cystic changes along inferior surface and gentle lobulations of superior surface. It showed multiple intralesional linear hyperintense and hypointense areas on T2WI, suggestive of vascular channels and fibrous septae respectively. Lipomatous component was well appreciated on Non-FatSat T1WI sequence. Few hypertrophied and tortuous vessels were also seen in the surrounding breast parenchyma, consistent with ultrasound findings.

d) Imaging diagnosis: The imaging findings demonstrated Breast Imaging-Reporting and Data System (BIRADS) 4A lesion. The primary impression considered phyllodes tumour and next best differential included giant fibroadenoma.

e) Therapeutic Intervention: Right breast lumpectomy with wide excision of margins to ensure adequate margins, without axillary lymph node dissection was performed. The tumour was found to be free from the overlying skin, nipple-areolar complex and the retro areolar ducts. The gross examination of the specimen confirmed it to be a well encapsulated mass with mildly bosselated superior margins. 
f) Histopathology revealed benign fibroepithelial tumor with an intracanalicular pattern and few cleft-like spaces (Figure 4). It showed overall low stromal cellularity with foci of adipose tissue within the tumor.

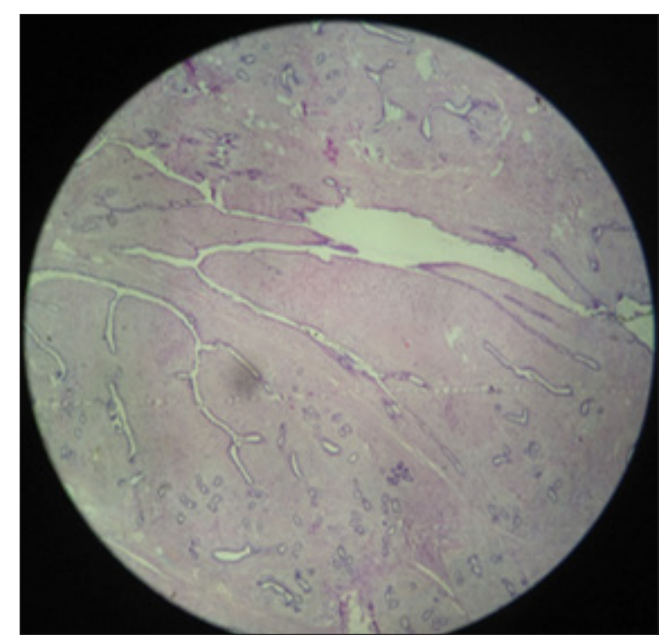

Figure 4: Histopathology revealed an intracanalicular pattern and few cleft-like spaces with overall low stromal cellularity and foci of adipose tissue.

g) Final Diagnosis: Giant Fibroadenoma type of benign fibroepithelial tumor.

\section{Discussion}

Fibroadenomas are most commonly seen breast lesions in adolescent girls and young women, which occasionally develop into very large masses (often more than $5 \mathrm{~cm}$ ); such masses are called juvenile giant fibroadenomas. These tumours are frequently cellular type of fibroadenomas and comprise 7-8 \% of all fibroadenoma subtypes [9]. Whereas phyllodes tumors are rare fibroepithelial tumors and demonstrate a wide spectrum of pathology from benign to malignant which may have the potential for infiltrating growth, recurrence, or metastasis in rare cases [3]. Both giant fibroadenoma and phyllodes tumor are fibroepithelial tumors and arise from intralobular stroma, hence share substantial overlap and similarity in physical, radiological and pathological features, specifically giant fibroadenomas and benign or borderline phyllodes tumors $[10,11]$.

Both tumors usually manifest as large, well-circumscribed, oval or lobulated masses on mammography $[5,7,12]$. However, few distinct features are differentiating, such as high density favours phyllodes whereas calcifications favour giant fibroadenoma $[7,13,14]$. The rounded cystic changes and cleft in otherwise solid mass lesion may favour the diagnosis of phyllodes tumors over fibroadenoma, but are not pathognomonic as they can also be seen in juvenile giant fibroadenoma $[5,7,12,14]$, as in our case. In spite of well-documented and well-established discriminating radiological features, routine breast radiographic evaluations can fail to differentiate giant fibroadenoma from phyllodes tumors in many cases $[15,16]$.
Our patient manifested with all the well documented radiological features of phyllodes tumor, including large size ( $\sim 12 \mathrm{~cm}$ in largest diameter), few cystic changes and clefts, increased vascularity and prominent surrounding vessels. But the histopathological features were suggestive of giant type of juvenile fibroadenoma. The therapeutic approaches for both the tumors are different, therefore it is critical for a surgeon to differentiate both the tumors preoperatively to decide the correct treatment plan, avoiding reoperation because of inadequate margins and to prevent surgical overtreatment as well $[5,11]$. Wide location excision with at least $1 \mathrm{~cm}$ margin is the treatment of choice for phyllodes tumor, however the margin is subject to individual interpretations. This is in contrast to treatment of fibroadenomas which are usually managed non-operatively or by simple enucleation. Thus, if a phyllodes tumor is misdiagnosed as fibroadenoma, it is likely to be inadequately resected thus increasing the likelihood of recurrence and re-excision which also leads to psychological morbidity to the patient [5].

\section{Conclusion}

Diagnostic discrimination between giant fibroadenoma and phyllodes tumor is still challenging. It is critical to keep in mind that both the tumors share substantial overlap of standard radiographic features and hence it is sometimes difficult to differentiate between them on basis of imaging alone and may lead to misdiagnosis in some exceptional cases.

\section{References}

1. Kalambo M, Adrada BE, Adeyefa MM, Krishnamurthy S, Hess K, et al. (2018) Phyllodes tumor of the breast: Ultrasound-pathology correlation. American Journal of Roentgenology 210(4): W173-W179.

2. Tummidi S, Kothari K, Agnihotri M, Naik L, Sood P (2020) Fibroadenoma versus phyllodes tumor: a vexing problem revisited. BMC Cancer 20(1): 648.

3. Duman L, Gezer NS, Balcı P, Altay C, Başara I, et al. (2016) Differentiation between phyllodes tumors and fibroadenomas based on mammographic sonographic and MRI features. Breast Care (Basel) 11(2): 123-127.

4. Abe M, Miyata S, Nishimura S, Iijima K, Makita M, et al. (2011) Malignant transformation of breast fibroadenoma to malignant phyllodes tumor: long-term outcome of 36 malignant phyllodes tumors. Breast Cancer 18(4): 268-272.

5. Jacklin RK, Ridgway PF, Ziprin P, Healy V, Hadjiminas D, Darzi A (2006) Optimising preoperative diagnosis in phyllodes tumour of the breast. J Clin Pathol 59(5): 454-459.

6. Dillon MF, Quinn CM, McDermott EW, Doherty A, Higgins N, et al. (2006) Needle core biopsy in the diagnosis of phyllodes neoplasm. Surgery 140(5): 779-784.

7. Yılmaz E, Sal S, Lebe B (2002) Differentiation of phyllodes tumors versus fibroadenomas. Mammographic and sonographic features. Acta Radiol 43(1): 34-39.

8. Feder JM, de Paredes ES, Hogge JP, Wilken JJ (1999) Unusual breast lesions: radiologic-pathologic correlation. Radiographics 19: 11-26.

9. Greydanus DE, Matytsina L, Gains M (2006) Breast disorders in children and adolescents. Prim Care 33(2): 455-502.

10. Park CA, David LR, Argenta LC (2006) Breast asymmetry: presentation of a giant fibroadenoma. Breast J 12(5): 451-461. 
11. Krishnamurthy S, Ashfaq R, Shin HJ, Sneige N (2000) Distinction of phyllodes tumor from fibroadenoma: A reappraisal of an old problem. Cancer 90(6): 342-349.

12. Bode MK, Rissanen T, Sarkkinen MA (2007) Ultrasonography and core needle biopsy in the differential diagnosis of fibroadenoma and tumor phyllodes. Acta Radiol 48(7): 708-713.

13. Foxcroft LM, Evans EB, Porter AJ (2007) Difficulties in the preoperative diagnosis of phyllodes tumours of the breast: a study of 84 cases. Breast 16(1): 27-37.
14. Chao TC, Lo YF, Chen SC, Chen MF (2002) Sonographic features of phyllodes tumors of the breast. Ultrasound Obstet Gynecol 20(1): 64-71.

15. Page JE, Williams JE (1991) The radiological features of phylloides tumour of the breast with clinicopathological correlation. Clin Radiol 44(1): 8-12.

16. Alhefdhi A (2017) Giant Fibroadenoma Mimicking Phylleoyeds Tumor. Obstet Gynecol Int J 6(4): 00213.

For possible submissions Click below: 